\title{
Le risque moral dans la relation de mandat
}

\section{Monsieur Jean-Jacques Laffont}

\author{
Abstract \\ Moral hazard in the principal-agent relationship
}

Good risk sharing is one of the goals that an economic organization must achieve. The ability that some agents have to modify risk by taking actions non observable by other agents, the so called moral hazard problem, makes this task difficult. The purpose of this paper is to give an overview of the main results obtained in the principal-agent model to characterize optimal risk sharing rules.

\section{Résumé}

Le risque moral dans la relation de mandat

Un bon partage des risques est une des conditions importantes que se doit de réaliser une organisation économique. Or l'aptitude qu'ont certains agents d'altérer les risques par des actions non observables par les autres parties que l'on a appelé un peu maladroitement problème de risque moral ou de hasard moral rend plus délicate la recherche de ce partage. L'objectif de cette note est de faire un tour d'horizon des résultats essentiels obtenus sur ce problème dans le contexte du modèle principalagent.

\section{Citer ce document / Cite this document :}

Laffont Jean-Jacques. Le risque moral dans la relation de mandat. In: Revue économique, volume 38, n¹, 1987. pp. 5-24;

http://www.persee.fr/doc/reco_0035-2764_1987_num_38_1_408967

Document généré le 28/05/2016 


\title{
Le risque moral dans la relation de mandat
}

\author{
Jean-Jacques Laffont
}

\begin{abstract}
Un bon partage des risques est une des conditions importantes que se doit de réaliser une organisation économique. Or l'aptitude qu'ont certains agents d'altérer les risques par des actions non observables par les autres parties que l'on a appelé un peu maladroitement problème de risque moral ou de hasard moral rend plus délicate la recherche de ce partage. L'objectif de cette note est de faire un tour d'horizon des résultats essentiels obtenus sur ce problème dans le contexte du modèle principal-agent.
\end{abstract}

L'organisation la plus simple que l'on puisse imaginer est sans doute celle qui met en jeu deux parties, un employeur et un ouvrier, un propriétaire terrien et un fermier, un avocat et son client, une compagnie d'assurances et un assuré, un manager d'entreprise et l'Etat ou l'assemblée des actionnaires, etc. Souvent, l'une des parties, le mandant ou principal, délègue au mandataire ou agent une action qui influence son bien-être, représenté par la production pour un employeur, la récolte pour le propriétaire terrien, la nature du jugement pour le client de l'avocat, etc. Si l'action de l'agent est observable, le principal peut contrôler parfaitement l'agent, sous réserve qu'il ait le pouvoir d'appliquer les pénalités nécessaires pour punir tout comportement déviant de l'agent.

En général, l'action de l'agent (niveau d'effort par exemple) n'est qu'imparfaitement observable. En effet, le résultat observé par le principal est le produit joint d'une action que seul connaît l'agent et d'un aléa (que l'agent connaît ou ne connaît pas au moment où il décide de son action). Le produit réalisé pour le compte du principal par l'agent est donc aléatoire ; il dépend positivement d'une variable d'action de l'agent, non observable par le principal et coûteuse pour l'agent. Le problème essentiel de cette mini-organisation est de définir des règles de partage du produit.

Nous savons, depuis Arrow [1953], Borch [1962], que le partage d'un revenu risqué entre agents économiques est réalisé de façon Pareto

* Ce travail a bénéficié du soutien du Commissariat général du Plan. Je remercie les rapporteurs pour leurs commentaires constructifs. 
optimale ex-ante lorsque les taux marginaux de substitution entre le revenu dans les différents états de la nature sont égalisés entre les agents économiques. En particulier, si le principal est neutre envers le risque, alors que l'agent a de l'aversion pour le risque, l'agent doit avoir un revenu constant pour être complètement assuré par le principal. Ce partage du risque Pareto optimal a un inconvénient majeur : l'agent n'a plus aucune incitation à réaliser d'effort, puisque son revenu n'en dépend pas. Faire dépendre le revenu de l'agent du produit fait s'écarter du partage des risques Pareto optimal.

L'objectif de cette note est de montrer de façon assez détaillée comment doivent être construits les contrats optimaux de cette organisation ${ }^{1}$ en présence de ce qu'on a appelé le risque moral, le hasard moral ou l'aléa de moralité ${ }^{2}$, qui est cette combinaison de non-observabilité de l'action et d'aléa sur le produit qui rend difficile l'interprétation du produit en termes d'action.

Dans une première section, nous exposons les résultats économiques les plus importants obtenus par une approche dite du premier ordre qui néglige les conditions du second ordre du problème de maximisation de l'agent. La deuxième section donne des conditions suffisantes sous lesquelles cette approche est valable et rend compte des tentatives pour la dépasser. La troisième section étudie dans quelle mesure la concurrence entre plusieurs agents peut être exploitée par le principal. Enfin, la répétition de la relation principal/agent ouvre de nouvelles possibilités de contrats qui sont exposées dans la dernière section. La conclusion indique les voies de recherche actuellement explorées.

\section{L'APPROCHE DU PREMIER ORDRE}

Soit $\tilde{y}$ une variable aléatoire qui représente le revenu brut du principal, par exemple l'output du processus de production possédé par le principal. La distribution de probabilité de $\ddot{y}$ est affectée par une variable $a$ contrôlée par un agent et non observable par le principal; $a$ est, par exemple, le niveau d'effort de l'agent. Soit $\mathrm{F}(y, a)$ la fonction de répartition de $\bar{y}$ et $f(y, a)$ sa densité de probabilité. Nous supposons que le support de cette distribution est indépendant de $a$.

1. La littérature a beaucoup tàtonné avant de parvenir à une formulation satisfaisante de la solution de ce problème (voir Simon [1951], Arrow [1963], Pauly [1968], Zeckhauser [1970], Spence et Zeckhauser [1971], Stiglitz [1971], Ross [1973), Mirrlees [1975], Harris et Raviv [1979], Shavell [1979], Holmström [1979]).

2. Le qualificatif de moral est maladroit mais peut s'expliquer de la façon suivante. L'agent pourrait s'engager moralement à réaliser une action déterminée en commun; c'est bien alors l'incertitude que le principal a sur la moralité de l'agent qui créerait le problème. 
Le problème posé est celui de la détermination de la compensation $t$ (exprimée en unités de $y$ ) offerte à l'agent pour le dédommager de la désutilité de son action $a$. Nous supposons que la fonction d'utilité de l'agent est séparable :

$$
\mathrm{V}(t, a)=v(t)-w(a)
$$

avec

$$
v^{\prime}>0, v^{\prime \prime} \leq 0 ; w^{\prime}>0, w^{\prime \prime}>0
$$

Pour assurer la participation de l'agent, il faut lui donner en espérance mathématique un niveau d'utilité supérieur à ce qu'il pourrait obtenir en dehors de la relation avec le principal. Ce niveau d'utilité appelé niveau de rationalité individuelle de l'agent, ici normalisé à zéro, est exogène; c'est en cela que l'analyse a un caractère d'équilibre partiel ${ }^{1}$.

$$
\mathrm{EV}(t, a) \geq 0
$$

L'utilité du principal est alors :

$$
\mathrm{U}(y-t), \quad \mathrm{U}^{\prime}>0, \quad \mathrm{U}^{\prime \prime} \leq 0
$$

Supposons, comme préliminaire, que le principal observe et donc contrôle $a$. Il peut choisir le niveau de $a$ et déterminer la fonction $t(\tilde{y})$ qui partage au mieux les risques. Son programme d'optimisation s'écrit :

T.Q.

$$
\underset{(a, t(.))}{\operatorname{Max}} \operatorname{E} U(\tilde{y}-t(\tilde{y}))
$$

$$
\mathrm{E} v(t(\tilde{y}))-w(a) \geq 0
$$

Le lagrangien s'écrit :

$$
\mathrm{L}=\int \mathrm{U}(y-t(y)) f(y, a) d y+\lambda\left[\int v(t(y)) f(y, a) d y-w(a)\right]
$$

Les conditions du premier ordre sont :

$$
\begin{gathered}
\frac{U^{\prime}(y-t(y))}{v^{\prime}(t(y))}=\lambda \text { pour tout } y \\
f[U(y-t(y))+\lambda v(t(y))] f_{a}(y, a) d y=\lambda w^{\prime}(a)
\end{gathered}
$$

(7) peut se réécrire

$$
\frac{U^{\prime}(y-t(y))}{U^{\prime}\left(y^{\prime}-t\left(y^{\prime}\right)\right)}=\frac{v^{\prime}(t(y))}{v^{\prime}\left(t\left(y^{\prime}\right)\right)} \text { pour tout } y, y^{\prime}
$$

1. Voir Helpman et Laffont [1975] et Hellwig [1983] pour une étude des conséquences du risque moral en équilibre général avec contrats a linéaires $n$ sur le marché des assurances. 
c'est-à-dire l'égalité des taux marginaux de substitution du revenu entre les différents états de la nature caractérisés ici par la valeur de $y$. Il y a donc partage optimal du risque. (8) exprime qu'il faut égaliser la désutilité marginale sociale de $a$ et son utilité marginale sociale calculées avec un poids $\lambda$ de l'agent. Ce poids est ici endogène et tel que la contrainte (5) soit saturée. Si à la fois l'agent et le principal ont de l'aversion pour le risque $v^{\prime \prime}<0, u^{\prime \prime}<0$ ), on observe en différentiant (7) que le revenu de l'agent et le revenu du principal augmentent strictement avec $y$.

Supposons maintenant que $a$ n'est pas observable par le principal. Celui-ci sait toutefois que, face à un système de transfert $t(\cdot)$, l'agent choisi $a$ dans son meilleur intérêt, c'est-à-dire :

$$
a \in \arg \max _{a^{\prime}} \int\left[v(t(y))-w\left(a^{\prime}\right)\right] f(y, a) d y^{1}
$$

A la contrainte de rationalité individuelle (5) s'ajoute maintenant (10).

L'approche du premier ordre consiste à remplacer (10) par la condition du premier ordre de ce programme de maximisation et à supposer que cette condition définit une solution unique. Le programme d'optimisation du principal s'écrit alors :

T.Q.

$$
\underset{(a, t(.))}{\operatorname{Max}} \int U(y-t(y)) f(y, a) d y
$$

$$
\begin{array}{ll}
f[v(t(y))-w(a)] f(y, a) d y \geq 0 & (\lambda) \\
\int \vee(t(y)) f_{a}(y, a) d y-w^{\prime}(a)=0 & (\mu)
\end{array}
$$

Nous avons supposé que le support de la distribution de $\tilde{y}$ est indépendant de $a$. Sinon il existe une manière triviale de réaliser l'allocation d'information parfaite en prenant des pénalités assez élevées. Intuitivement, si l'agent ne réalise pas l'action $a^{*}$ spécifiée par le principal, il existe une probabilité non nulle qu'il soit identifié, car le support de $\tilde{y}$ n'est pas ce qu'il devrait être. Il suffit alors de prendre dans ces cas d'identification une pénalité assez forte pour que l'agent ne se hasarde pas à une action différente de $a^{*}$. On peut donc obtenir l'optimum.

Les conditions du premier ordre du problème (11) s'écrivent :

$$
\begin{gathered}
\frac{\mathrm{U}^{\prime}(y-t(y))}{v^{\prime}(t(y))}=\lambda+\mu \frac{f_{a}(y, a)}{f(y, a)} \\
f \mathrm{U}(y-t(y)) f_{a}(y, a) d y+\mu\left[\int v(t(y)) f_{a a}(y, a) d y-w^{\prime \prime}(a)\right]=0
\end{gathered}
$$

L'action $a$ a un effet négatif bien défini sur l'utilité de l'agent. Pour qu'elle ait un effet positif bien défini sur l'output, nous supposons qu'un

1. $\arg \max _{a} \Phi(a)$ signifie "l'ensemble des valeurs de a qui maximisent la fonction $\Phi(\cdot)$ par rapport à $a$ ". 
accroissement de $a$ produit une amélioration de $\bar{y}$ au sens de la dominance stochastique du premier ordre ${ }^{1}$ ou :

$$
\mathrm{F}_{a}(y, a) \leq 0
$$

(avec une inégalité stricte pour un ensemble de probabilité positive).

On peut alors montrer que lorsque l'agent a de l'aversion au risque, le partage des risques n'est pas Pareto optimal, c'est-à-dire $\mu>0$. En effet, en s'écartant du partage Pareto optimal, le principal peut inciter l'agent à un meilleur niveau d'effort.

Théorème : Holmström [1979], Shavell [1979]

Sous les hypothèses ci-dessus, si de plus $v^{\prime \prime}<0$, alors $\mu>0$.

Démonstration. Supposons, au contraire, $\mu \leq 0$. D'après la condition du premier ordre (14), on a, en posant $r(y)=y-t(y)$ :

$$
\frac{U^{\prime}(r(y))}{v^{\prime}(y-r(y))}=\lambda+\mu \frac{f_{a}(y, a)}{f(y, a)}
$$

$r(y)$ est le revenu du principal à l'optimum du problème d'information imparfaite. Soit $r_{\lambda}(y)$ son revenu en information parfaite lorsque le multiplicateur de la contrainte de rationalité individuelle est $\lambda$ [voir (7)].

Alors pour $y \in\left\{y \mid f_{a}(y, a) \supseteq 0\right\}$

$$
\frac{U^{\prime}(r(y))}{v^{\prime}(y-r(y))} \leq \lambda=\frac{U^{\prime}\left(r_{\lambda}(y)\right)}{v^{\prime}\left(y-r_{\lambda}(y)\right)}
$$

Donc, comme $U^{\prime \prime} \leqslant 0$ et $v^{\prime \prime}<0, r_{\lambda}(y) \leqslant r(y)$.

De même, on montre que pour $y \in\left\{y \mid f_{a}(y, a) \leq 0\right\} r_{\lambda}(y) \supseteq r(y)$. Par conséquent :

$$
\int \cup(r(y)) f_{a}(y, a) d y \geq \int \cup\left(r_{\lambda}(y)\right) f_{\alpha}(y, a) d y
$$

Montrons que le membre de droite de (19) est strictement positif en posant $[\alpha, \beta]$ le support de $\tilde{y}$

$$
\begin{aligned}
\int_{\alpha}^{\beta} \mathrm{U}\left(r_{\lambda}(y)\right) f_{a}(y, a) d y & =\left.\mathrm{U}\left(r_{\lambda}(y)\right) F_{\alpha}(y, a)\right|_{\alpha} ^{\beta} \\
& -f_{\alpha}^{\beta} F_{a}(y, a) \mathrm{U}^{\prime}\left(r_{\lambda}(y)\right) r_{\lambda}^{\prime}(y) d y
\end{aligned}
$$

Comme $\mathrm{F}(\beta, a)=1$ pour tout $a, \mathrm{~F}_{a}(\beta, a)=0$ pour tout $a$

$\mathrm{F}(\alpha, a)=0$ pour tout $a, \mathrm{~F}_{\alpha}(\alpha, a)=0$ pour tout $a$.

Par ailleurs, $U^{\prime}>0$ et $r_{\lambda}^{\prime}>0$ d'après la remarque suivant (9). Comme $\mathrm{F}_{a}(y, a)$ est strictement négatif pour un ensemble de probabilité positive,

1. Faire un dessin pour comprendre (16). 
nous avons :

$$
\int \cup(r(y)) f_{a}(y, a) d y>0
$$

Considérons maintenant la deuxième condition du premier ordre (15). Comme le niveau d'action choisi est un maximum (intérieur) du programme d'optimisation de l'agent, le coefficient de $\mu$ (qui est la dérivée seconde de ce programme) est négatif ou nul. Alors d'après (15) et (21), nous devons avoir $\mu>0$ une contradiction. CQFD.

Pour commenter ce résultat, prenons le cas où le principal n'a pas d'aversion au risque $\left(U^{\prime \prime}=0\right)$. Le partage optimal du risque nécessiterait une assurance complète de l'agent. Mais alors son revenu serait indépendant de son action et il choisirait l'action qui lui est la plus favorable, un niveau d'effort nul dans l'interprétation effort de cette variable. Pour l'amener à choisir un niveau d'effort plus favorable au principal, on fait dépendre son revenu de la seule variable observable, l'output y. Mais celui-ci est aléatoire, donc il ne peut pas y avoir partage optimal du risque.

Remarquons que, si l'agent n'a pas d'aversion au risque, l'optimum d'information parfaite est obtenu en donnant à l'agent $\tilde{y}$-constante (c'est-àdire le principal a un revenu fixe) où la constante est déterminée pour saturer la contrainte de rationalité individuelle. Comme l'agent reçoit tout le bénéfice de son action, son niveau d'action est optimal.

On s'attend intuitivement à ce que le transfert reçu par l'agent croisse avec $y$. Ceci peut être démontré en différentiant (14) sous l'hypothèse supplémentaire selon laquelle $\frac{\partial f}{\partial a}(y, a) / f(y, a)$ est une fonction croissante de $y^{1}$. Cette condition appelée condition du rapport de vraisemblance monotone $(\mathrm{VM})$ a l'interprétation intuitive suivante. $\log f(y, a)$ est la vraisemblance du modèle où $y$ est la variable endogène et $a$ le paramètre à estimer. Plus cette valeur est grande pour un $\left(y, a^{*}\right)$ donné, plus cette valeur de $y$ suggère que la vraie valeur de $a$ est bien $a^{*}$. Donc $\frac{\partial f}{\partial a}\left(y, a^{*}\right) / f\left(y, a^{*}\right)=\frac{\partial}{\partial a} \log f\left(y, a^{*}\right)$ mesure de combien on est incliné à penser que la valeur $y$ observée ne vient pas d'un modèle avec une valeur $a^{*}$. La condition du premier ordre (14) exprime qu'on doit accepter

1. Cette condition peut aussi s'exprimer sous la forme $: \frac{f\left(y, a_{2}\right)}{f\left(y, a_{1}\right)}$ est croissante en $y$ dès que $a_{1}<a_{2}$; autrement dit, l'observation de valeurs plus élevées de $y$ rend plus plausible que l'action ait été $a_{2}$. plutôt que $a_{1}$. Noter que $\frac{f\left(y, a_{2}\right)}{f\left(y, a_{1}\right)}$ croissant en $y$ est équivalent $\left(\frac{f\left(y, a_{2}\right)}{f\left(y, a_{1}\right)}-1\right) /\left(a_{2}-a_{1}\right)=\frac{\frac{f\left(y, a_{2}\right)-f\left(y, a_{1}\right)}{a_{2}-a_{1}}}{f\left(y, a_{1}\right)}$ croissant en $y$ qui est la version discrète de l'hypothèse du tẹxte. 
des déviations par rapport au premier rang d'autant plus grandes que cette mesure est grande. En effet $y$ est utilisé comme un signal informatif sur la valeur de $a$ choisie par l'agent. Si le signal est peu informatif (vraisemblance $\log f(y, a)$ très plate en $a)$ on ne peut guère espérer inciter à beaucoup d'effort et il vaut mieux essayer de bien assurer l'agent pour lui donner son niveau de rationalité individuelle.

Est-il utile de tenir compte d'autres variables $z$ dont la distribution dépend de $a$ pour mieux contrôler l'agent par un transfert fonction à la fois de $y$ et de $z$ ? Holmström [1979] montre le résultat intuitif selon lequel si $y$ n'est pas une statistique suffisante pour le couple $(y, z)$ par rapport à $a$, l'observation de $z$ a de la valeur pour le principal et le transfert optimal dépend de $y$ et $z$.

Montrons comment une telle théorie permet de justifier la coassurance. Pour cela, considérons un agent qui peut avoir un accident avec probabilité $1-f(0, a)$. La perte $x$ qu'il subit, s'il a un accident, est variable et soit $f(x, a)$ la densité de probabilité de cette perte avec $\int f(x, a) d x=1-f(0, a)$. Le principal est ici la compagnie d'assurances et $x$ joue le rôle de $-y$ ci-dessus. L'agent diminue la probabilité d'accident avec son action $\frac{\partial f}{\partial a}(0, a)>0$ et diminue la probabilité d'une perte de taille $x, x+d x$ pour tout $x, \frac{\partial f}{\partial a}(x, a)<0$, ce qui correspond à l'hypothèse de dominance stochastique du premier ordre dans ce contexte plus général où la distribution de $\tilde{y}$ a un atome (c'est-à-dire une masse positive de probabilité) en 0 . Considérons la condition du premier ordre (14). Comme le membre de gauche de (14) est continu en $t$ et que le membre de droite a une discontinuité en $x=0, t(x)$ doit avoir une discontinuité en $x=0$. De plus, en comparant avec la condition du premier ordre d'information parfaite, on voit que $t(0)>t(x)$ pour tout $x$. Donc

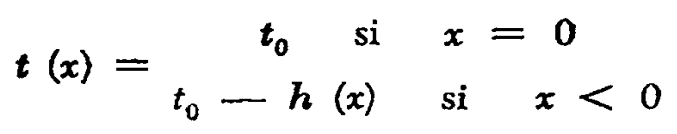

$t_{0}$ est le revenu de l'agent s'il n'a pas d'accident, c'est-à-dire après paiement seulement de la prime. S'il a un accident il doit partager avec la compagnie la perte due à l'accident; il y a donc coassurance. Ce partage du risque non optimal si l'agent a de l'aversion au risque le conduit à un comportement plus prudent. Noter enfin que si l'action n'affecte que la probabilité d'accident (et pas la densité de la perte si accident), $h(x)$ est constant. On a alors une justification de la franchise différente de celle donnée par Arrow [1963] qui reposait sur l'existence d'un coût administratif. 


\section{VALIDITÉ ET LIMITES DE L'APPROCHE DU PREMIER ORDRE}

L'approche du premier ordre consiste à remplacer la contrainte :

$$
a \in \arg \max _{a} \mathrm{E} V\left(t(y), a^{\prime}\right)
$$

par la condition du premier ordre de ce programme de maximisation, c'est-à-dire par la contrainte

$$
\mathrm{E} \frac{\partial \mathrm{V}}{\partial a}(t(y), a)=0
$$

Portons symboliquement $-t$ en abscisse et $a$ en ordonnée (voir graphique). Représentons par la courbe $\mathbf{A A}^{\prime}$ l'ensemble des a qui satisfait la condition du premier ordre. Comme à $t$ donné l'agent cherche à minimiser $a$, seule la partie de la courbe en trait gras identifie les vrais maximas.

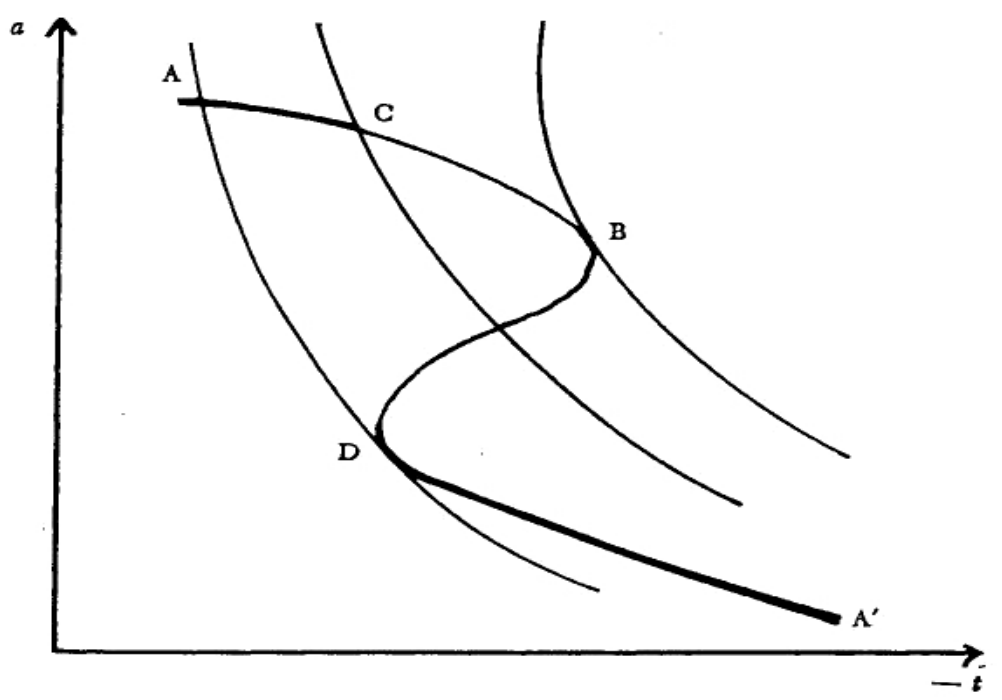

Traçons aussi les courbes d'indifférence du principal. Si le principal maximise sous la condition du premier ordre, c'est-à-dire dans $\mathbf{A A}^{\prime}$ il obtient $B$ alors que le vrai optimum est $C$. Non seulement $B$ n'est pas l'optimum, mais $C$ ne satisfait pas les conditions du premier ordre (seuls $\mathrm{B}$ et $\mathrm{D}$ le font).

Le problème d'optimisation de l'agent n'est pas toujours concave en $a$ et donc la condition du premier ordre ne caractérise pas toujours l'optimum (voir Mirrlees [1975] et Guesnerie et Laffont [1978]). On peut, dès lors, soit rechercher des conditions suffisantes qui valident l'approche du premier ordre, soit adopter une approche plus générale.

Mirrlees [1975] et Rogerson [1985 b] ont donné des conditions suffisantes. Une démonstration du caractère suffisant de ces conditions 
peut être esquissée lorsqu'on sait que la solution optimale est une des solutions des conditions du premier ordre du principal. D'après la section précédente on sait que sous l'hypothèse $(\mathrm{VM}), t(y)$ est croissante. Le problème sera concave si :

$$
\int[v(t(y))-w(\mathrm{a})] f_{a a}(\mathrm{y}, a) d \mathrm{y}-\mathrm{w}^{\prime \prime}(a) \leq 0
$$

En intégrant par partie, nous obtenons

$$
-\int F_{a a}(y, a) v^{\prime}(t(y)) t^{\prime}(y) d y-w^{\prime \prime}(a) \leq 0
$$

Puisque $w^{\prime \prime}>0$, l'hypothèse de convexité en $a$ de la fonction de répartition suffit avec (VM) à assurer la concavité en $a$ du problème (voir Rogerson [1985 b] pour une démonstration plus générale).

Que faire lorsque F n'est pas convexe en $a$ ? Grossman et Hart [1983] ont essayé de progresser en décomposant le programme du principal en deux étapes. Ils considèrent un cadre discret avec un nombre fini de valeurs de $y, y_{1}, \ldots, y_{n}$ et un principal neutre envers le risque.

Pour chaque action $a$ dans l'ensemble $A$ des actions admissibles, le principal peut calculer le coût espéré $C(a)$ qu'il lui faut subir pour amener l'agent à choisir $a$. Dans une deuxième étape, le principal choisit l'action qu'il préfère en comparant les bénéfices $\mathrm{B}(a), a \in \mathrm{A}$ et les coûts $\mathrm{C}(a), a \in \mathrm{A}$. L'avantage de cette démarche est que le premier problème est assez simple, car il peut être réécrit comme un problème de minimisation d'une fonction convexe dans un ensemble convexe.

Soit $t_{1}, \ldots, t_{n}$ le vecteur de transferts associé à l'observation de $y_{1}, \ldots, y_{n}$. Soit $\pi_{i}(a)$ ( $>0$ pour tout $a \in \mathrm{A}$ ) la probabilité de $y_{i}$, si l'agent choisit $a$, $i=1, \ldots, n$. Pour amener l'agent à choisir $a$, cela coûte $C(a)$ la valeur du maximande à l'optimum du programme suivant :

$$
\operatorname{Min} \sum_{i=1}^{n} \pi_{i}(a) t_{i}
$$

T.Q.

$$
\begin{gathered}
\sum_{i=1}^{n} \pi_{i}(a)\left[v\left(t_{i}\right)-w(a)\right] \geq 0 \\
\sum_{i=1}^{n} \pi_{i}(a)\left[v\left(t_{i}\right)-w(a)\right] \geq \sum_{i=1}^{n} \pi_{i}\left(a^{\prime}\right)\left[v\left(t_{i}\right)-w\left(a^{\prime}\right)\right] ; \forall a^{\prime} \in \mathrm{A} .
\end{gathered}
$$

(27) est la contrainte de rationalité individuelle et (28) est l'ensemble des contraintes incitatives.

Posons $v\left(t_{i}\right)=v_{i}$ et $t_{i}=v^{-1}\left(v_{i}\right)$. Comme $v(\cdot)$ est concave, croissante $v^{-1}(\cdot)$ est convexe et le programme peut s'écrire :

$$
\operatorname{Min} \sum_{i=1}^{n} \pi_{i}(a) v^{-1}\left(v_{i}\right)
$$

T.Q. 


$$
\begin{gathered}
\sum_{i=1}^{n} \pi_{i}(a)\left[v_{i}-w(a)\right] \geq 0 \\
\sum_{i=1}^{n} \pi_{i}(a)\left[v_{i}-w(a)\right] \geq \sum_{i=1}^{n} \pi_{i}\left(a^{\prime}\right)\left[v_{i}-w\left(a^{\prime}\right)\right] \cdot \forall a^{\prime} \in \mathrm{A} .
\end{gathered}
$$

La fonction objectif est convexe en $\left(v_{1}, \ldots, v_{n}\right)$ et les contraintes linéaires. Soit $\lambda$ le multiplicateur de (30) et $\mu\left(a^{\prime}\right), a^{\prime} \in$ A les multiplicateurs de (31). Les conditions du premier ordre (nécessaires et suffisantes) s'écrivent en divisant par $\pi_{i}(a)$ :

$$
\frac{1}{v^{\prime}\left(t_{i}\right)}=\lambda+\underset{a^{\prime} \in \sum_{K}(a)}{\sum} \mu\left(a^{\prime}\right)\left(\frac{\pi_{i}(a)-\pi_{i}\left(a^{\prime}\right)}{\pi_{i}(a)}\right) i=1, \ldots, n
$$

où $\mathrm{K}(a)$ est l'ensemble des contraintes incitatives saturées.

La comparaison avec (14) montre que la différence avec l'approche du premier rang est que des contraintes incitatives autres que locales (c'est-à-dire des $a^{\prime}$ non voisins de $a$ ) peuvent être saturées d'où la somme $\underset{a^{\prime} \in \mathrm{K}(a)}{\sum_{\text {. }}}$.

En travaillant uniquement avec le programme (29), Grossman et Hart [1983] obtiennent certains résultats. Ils montrent l'équivalent du théorème 1 , c'est-à-dire le partage non optimal des risques quand l'agent a de l'aversion au risque. Ils montrent aussi que le revenu du principal et le revenu de l'agent ne peuvent être toujours négativement reliés et que le transfert ne peut ètre partout non croissant. Toutefois, pour montrer que le transfert est croissant en $y$, ils doivent faire des hypothèses qui valident l'approche du premier ordre.

Ainsi, en l'absence de conditions suffisantes très restrictives qui valident l'approche du premier ordre, nous avons très peu d'informations sur la solution optimale si ce n'est qu'elle implique un arbitrage entre encouragement à l'effort et bon partage des risques.

\section{CONCURRENCE ENTRE AGENTS}

Dans de nombreux contextes, le principal est en relation avec plusieurs agents, et la question se pose de savoir s'il peut utiliser la concurrence que se feront les agents entre eux. L'intuition de base est assez simple. Si l'output observable de l'agent $j$ n'est pas «informatif » sur l'effort de l'agent $i$, faire dépendre la rémunération de $i$ de l'output de $j$ ne peut qu'accroître l'aléa auquel fait face $i$ sans pour cela l'inciter à plus d'effort. Par contre, s'il est informatif, il faut alors déterminer l'arbitrage optimal entre incitation à l'effort et partage de risque dans 
le même esprit qu'à la section 1 . Il va sans dire que les problèmes liés à l'approche du premier ordre restent intacts.

Soit $\tilde{y}^{i}$ la variable aléatoire qui représente l'output dû à l'agent $i$, $i=1, \ldots$, I. La distribution jointe de $\bar{y}=\left(\tilde{y}^{1}, \ldots, \tilde{y}^{\mathrm{T}}\right)$ dépend des actions des agents $a=\left(a^{1}, \ldots, a^{\mathrm{I}}\right): \mathrm{F}(y, a)$ (densité $f(y, a)$ ). Le transfert $t^{i}$ que reçoit l'agent $i$ est maintenant fonction du vecteur des observables, c'est-àdire les outputs individuels $y^{1}, \ldots, y^{\mathrm{x}}, i=1, \ldots, \mathrm{I}$. Etant donné un système de transferts $\left(t^{i}(\cdot)\right)$, le choix d'actions des agents est représenté par un équilibre de Nash, c'est-à-dire :

$$
\begin{array}{r}
a^{* i} \in \arg \max _{a^{i}} \int\left[v^{i}\left(t^{i}(y)\right)-w^{i}\left(a^{i}\right)\right] d \mathrm{~F}\left(y, a^{i}, a^{*-i}\right) \\
i=1, \ldots, \mathrm{I}
\end{array}
$$

où

$$
\left(a^{i}, a^{*-i}\right)=\left(a^{* 1} \ldots a^{* i-1}, a^{i}, a^{* i+1}, \ldots, a^{* 1}\right) .
$$

Une première complication provient de la possibilité d'équilibres multiples que nous négligerons en supposant que les agents se coordonnent sur un équilibre particulier.

Le problème du principal peut alors s'écrire :

$$
\operatorname{Max}_{(t i(.))} f U\left(\sum_{i=1}^{x}\left(y^{i}-t^{i}(y)\right)\right) d F\left(y, a^{*}\right)
$$

T.Q.

$$
\begin{gathered}
\int\left[v^{i}\left(t^{i}(y)\right)-w^{i}\left(a^{*}\right)\right] d \mathrm{~F}\left(y, a^{*}\right) \geq 0 \\
a^{* i} \in \arg \max _{a^{i}} f\left[v^{i}\left(t^{i}(y)\right)-w^{i}\left(a^{i}\right)\right] d \mathrm{~F}\left(y, a^{i}, a^{*-i}\right) \\
i=1, \ldots, \mathrm{I}
\end{gathered}
$$

Une première justification pour le principal d'avoir des transferts $t^{t}$ dépendant de l'ensemble des $y^{i}$ est un souci d'assurance. Nous éliminons cette considération en supposant désormais que le principal est neutre envers le risque.

De façon générale, les transferts dépendent non linéairement de l'ensemble des observations $y^{1}, \ldots, y^{\mathbb{I}}$ et les résultats très limités des sections 1 et 2 peuvent être généralisés.

Mookherjee [1984] généralise l'approche de Grossman-Hart. Supposons qu'il y ait deux agents et soit

$$
\begin{gathered}
\pi_{i j}\left(a^{1}, a^{2}\right)=\operatorname{Pr}\left(\bar{y}^{1}=y_{i}^{1}, \bar{y}^{2}=y^{2}, / a^{1}, a^{2}\right) \\
i=1, \ldots, n \\
i=1, \ldots, n
\end{gathered}
$$

Avec des notations évidentes le programme de minimisation de coût (analogue à (26)) peut être écrit : 


$$
\begin{gathered}
\underset{\left(v_{i j}, v_{i j}^{2}\right)}{\operatorname{Min}} \sum_{i} \sum_{j} \pi_{i j}\left(a^{1}, a^{2}\right)\left\{\left(v^{1}\right)^{-1}\left(v_{i j}^{1}\right)+\left(v^{2}\right)^{-1}\left(v_{i j}^{2}\right)\right\} \\
\sum_{i} \sum_{j} \pi_{i j}\left(a^{1}, a^{2}\right)\left[v_{i j}^{1}-w^{1}\left(a^{1}\right)\right] \geq 0 \\
\sum_{i} \sum_{j} \pi_{i j}\left(a^{1}, a^{2}\right)\left[v_{i j}^{2}-w^{2}\left(a^{2}\right)\right] \geq 0
\end{gathered}
$$

$\sum_{i} \sum_{j} \pi_{i j}\left(a^{1}, a^{2}\right)\left(v_{i j}^{1}-w^{1}\left(a^{1}\right)\right) \geq \sum_{i} \sum_{j} \pi_{i j}\left(a^{\prime 1}, a^{2}\right)\left(v_{i j}^{1}-w^{1}\left(a^{\prime 1}\right)\right) \forall a^{\prime 1} \in A^{1}$ $\sum_{i} \sum_{j} \pi_{i j}\left(a^{1}, a^{2}\right)\left(v_{i j}^{2}-w^{2}\left(a^{2}\right)\right) \geq \sum_{i} \sum_{j} \pi_{i j}\left(a^{1}, a^{\prime 2}\right)\left(v_{i j}^{2}-w^{2}\left(a^{\prime 2}\right)\right) \forall a^{\prime 2} \in A^{2}$

En observant que les contraintes de rationalité individuelle sont saturées à l'optimum, le programme peut être réécrit :

$$
\underset{\left(v_{i j}^{1}, v_{i j}^{2}\right)}{\operatorname{Min}} \sum_{i} \sum_{j} \pi_{i j}\left(a^{1}, a^{2}\right)\left\{\left(v^{1}\right)^{-1}\left(v_{i j}^{1}\right)+\left(v^{2}\right)^{-1}\left(v_{i j}^{2}\right)\right\}
$$

T.Q.

$$
\begin{aligned}
& \sum_{i} \sum_{j} \pi_{i j}\left(a^{1}, a^{2}\right)\left[v_{i j}^{1}-w^{1}\left(a^{1}\right)\right]=0 \quad\left(\lambda^{1}\right) \\
& \sum_{i} \sum_{j} \pi_{i j}\left(a^{1}, a^{2}\right)\left[v_{i j}^{2}-w^{2}\left(a^{2}\right)\right]=0 \quad\left(\lambda^{2}\right) \\
& \sum_{i} \sum_{j} \pi_{i j}\left(a^{1}, a^{\prime 2}\right)\left[v_{i j}^{2}-w^{1}\left(a^{\prime 1}\right)\right] \leq 0 \quad\left(\mu^{1}\left(a^{\prime 1}\right)\right) \quad \forall a^{\prime 1} \in A^{1} \\
& \sum_{i} \sum_{j} \pi_{i j}\left(a^{\prime 1}, a^{2}\right)\left[v_{i j}^{2}-w^{2}\left(a^{\prime 2}\right)\right] \leq 0 \quad\left(\mu^{2}\left(a^{\prime 2}\right)\right) \quad \forall a^{\prime 2} \in A^{y}
\end{aligned}
$$

Les conditions du premier ordre de ce problème convexe s'écrivent :

$$
\begin{aligned}
& \frac{1}{v^{\prime 1}\left(t_{i j}\right)}=\lambda^{1}-a_{a^{\prime 1}} \sum_{\mathrm{A} 1} \mu^{1}\left(a^{\prime 1}\right) \frac{\pi_{i j}\left(a^{\prime 2}, a^{2}\right)}{\pi_{i j}\left(a^{1}, a^{2}\right)} \\
& \frac{1}{v^{\prime 2}\left(t_{i j}\right)}=\lambda^{2}-a_{a^{2}} \sum_{\mathrm{A}^{2}} \mu^{2}\left(a^{\prime 2}\right) \frac{\pi_{i j}\left(a^{1}, a^{\prime 2}\right)}{\pi_{i j}\left(a^{1}, a^{2}\right)}
\end{aligned}
$$

Nous dirons que, étant donné $a^{2} \in \mathbf{A}^{2}, y^{2}$ est un signal non informatif sur $a^{1}$ si

$$
\pi_{t j}\left(a^{\prime 1}, a^{2}\right)=h\left(y_{i}^{1}, y_{j}^{2}, a^{2}\right) \cdot f\left(y_{i}^{1}, a^{\prime 1}, a^{2}\right) \text { pour tout } a^{\prime 1} \in \mathbf{A}^{1}
$$

En d'autres termes, $y^{1}$ est une statistique suffisante pour le couple $y^{1}, y^{2}$ par rapport à $a^{1}$.

Alors

$$
\frac{\pi_{i j}\left(a^{\prime 1}, a^{2}\right)}{\pi_{1 j}\left(a^{1}, a^{2}\right)}=\frac{f\left(y_{i}^{1}, a^{\prime 1}, a^{2}\right)}{f\left(y_{i}^{1}, a^{1}, a^{2}\right)}
$$

et donc, d'après les conditions du premier ordre, $t_{i j}$ ne dépend pas de $j$. 
Donc, si étant donné $a^{2}$, la connaissance de $y^{2}$ ne donne pas d'information sur les vraisemblances relatives des valeurs de $a^{1}$, il est inutile d'utiliser l'observation de $y^{2}$ dans la rémunération de l'agent 1.

Par exemple si

$$
\begin{aligned}
& \tilde{y}^{1}=r\left(a^{1}\right)+\overline{\varepsilon^{1}} \\
& \tilde{y}^{2}=r\left(a^{2}\right)+\overline{\varepsilon^{2}}
\end{aligned}
$$

où $\tilde{\varepsilon}^{1}$ et $\tilde{\varepsilon}^{2}$ sont des variables aléatoires indépendantes, il ne sert à rien de vouloir utiliser les performances de l'un pour contrôler l'autre.

Par contre si à ces bruits idiosyncratiques $\tilde{\varepsilon}^{1}$ et $\tilde{\varepsilon}^{2}$ s'ajoute un bruit commun $\ddot{\varepsilon}$, alors en général $\bar{y}^{2}$ est informatif sur $a^{1}$. Intuitivement, si $y^{2}$ est grand, cela est dû avec une certaine probabilité au fait que $\tilde{\varepsilon}$ est grand; donc $y^{1}$ doit être assez grand même avec un effort $a^{1}$ faible.

$\mathrm{Si} \tilde{\varepsilon}^{1}$ et $\tilde{\varepsilon}^{2}$ sont parfaitement corrélés, alors il est possible d'obtenir l'optimum d'information parfaite, du moins tant que les agents ont un comportement de Nash (donc non coopératif) entre eux. S'écarter de l'action recommandée par le principal crée en effet une probabilité non nulle que ceci soit identifié à la lumière de l'output de l'autre agent. Une pénalité suffisante permet donc d'éviter ce comportement.

Au-delà de ces considérations générales sur les mécanismes optimaux, la littérature s'est beaucoup intéressée à une classe particulière de contrats, les tournois (Lazear et Rosen [1981], Green et Stockey [1983], Stiglitz et Nalebuff [1983]). Dans un tournoi, les rémunérations des agents dépendent uniquement de l'ordre des performances $y^{1}, \ldots, y^{\underline{x}}$. Il est évident que ces mécanismes ne sont pas optimaux en général. Par rapport à l'exemple ci-dessus, l'intérêt du tournoi est d'éliminer dans la rémunération la variabilité due au bruit commun $\tilde{\varepsilon}$, variabilité qui existerait dans un contrat non linéaire dépendant uniquement de la performance de l'agent lui-mềme. L'inconvénient par rapport à ces contrats non linéaires «indépendants» est l'accroissement de la variabilité due aux bruits idiosyncratiques des autres agents.

Toutefois, comme l'ont montré Green et Stockey [1983], lorsque le nombre d'agents est grand, l'ordre des performances devient un estimateur très précis des outputs des agents nets des bruits communs. On comprend alors qu'ils soient presque optimaux. 


\section{LA RÉPÉTITION DE LA RELATION PRINCIPAL-AGENT}

Dans de nombreuses circonstances, la relation principal-agent est de longue durée (assurance-assuré ; actionnaires-manager, ...). Il semble alors intuitif que, dans un modèle stationnaire, l'observation répétée de l'output $y_{t}$ permet par la loi des grands nombres d'éliminer le bruit et d'identifier avec une probabilité aussi proche de 1 que l'on désire une déviation de l'action recommandée par le principal. En choisissant une pénalité assez forte en cas de déviation on peut donc convaincre l'agent de choisir n'importe quelle action qui lui assure un niveau d'utilité supérieur à son niveau de rationalité individuelle, en particulier l'optimum d'information parfaite.

Cette intuition a été formalisée en utilisant la loi du logarithme itéré qui donne une mesure de la vitesse de convergence de la moyenne empirique d'une suite de variables indépendantes identiquement distribuées vers la moyenne (Rubinstein [1979], Radner [1981], Rubinstein et Yaari [1983]).

Ici, la variable aléatoire $\tilde{y}_{t}$ représente le dommage subi par l'agent à la date $t$. Son utilité sans assurance est

$$
\lim _{T \rightarrow \infty} \frac{1}{T} \sum_{t=1}^{T} \int\left[v\left(-y_{t}\right)-w\left(a_{t}\right)\right] f\left(y_{t}, a_{t}\right) d y_{t}{ }^{1}
$$

où $a_{t}$ est le niveau d'effort qu'il choisit à la date $t$ et qui influence la distribution des dommages.

Le principal est la compagnie d'assurances qui n'a pas d'aversion pour le risque. L'optimum d'information parfaite est donc l'assurance totale et un niveau d'action $a^{*}$ défini par

$$
\operatorname{Max}_{a}\left\{v\left(-\int y_{t} f\left(y_{t}, a\right) d y_{t}\right)-w(a)\right\}
$$

La prime d'assurance équitable est l'espérance du dommage :

$$
\Pi^{*}=\int y_{t} f\left(y_{t}, a\right) d y_{t}
$$

Le niveau d'utilité de l'agent est donc :

$$
\lim _{T \rightarrow \infty} \frac{1}{T} \sum_{t=1}^{T}\left[v\left(-\Pi^{*}\right)-w\left(a^{*}\right)\right]
$$

et le niveau d'utilité espéré de la compagnie d'assurances est nul ; son niveau d'utilité tend avec la probabilité 1 vers zéro, quand $T$ tend vers l'infini. du futur.

1. On utilise le critère de la limite de la moyenne. Il n'y a donc pas d'escompte 
En présence de risque moral, Rubinstein et Yaari [1983] montrent que la police d'assurance suivante réalise cet optimum d'information parfaite.

A chaque date $t$, la prime payée par l'agent est

- soit la prime équitable $\Pi^{*}$ correspondant au niveau d'action $a^{*}$ si le dossier de l'agent est satisfaisant, c'est-à-dire si le dommage moyen jusqu'à la date $t$ ne s'écarte pas trop de $\Pi^{*}$ ou encore :

$$
\frac{1}{t} \sum_{s=1}^{t} y_{s}<\Pi^{*}+\alpha^{t}
$$

avec

$$
\begin{aligned}
& \alpha^{t}=\sqrt{\frac{2 \lambda \sigma^{2} \log \log t}{t}} \\
& \lambda>1, \sigma^{2}=\operatorname{Var} \tilde{y}_{t} / a^{*}
\end{aligned}
$$

- soit une prime $\Pi^{*}$ donnant un niveau d'utilité instantané inférieur au niveau de rationalité individuelle si le dossier n'est pas satisfaisant.

Avec une telle politique, avec probabilité 1, l'agent n'est pénalisé qu'un nombre fini de fois s'il choisit $a=a^{*}$, alors qu'il serait pénalisé un nombre infini de fois s'il choisissait $a<a^{*}$. Le choix de la suite $\alpha^{z}$ est délicat (bien que toute suite convergeant vers zéro et supérieure à celle choisie conviendrait). D'une part, $\alpha^{t}$ doit converger vers zéro pour assurer que l'agent ne prend pas un niveau d'action inférieur à $a^{*}$. D'autre part, $\alpha^{t}$ ne doit pas converger trop vite vers zéro car, sinon, l'agent se verrait, en choisissant $a^{*}$, pénalisé trop souvent et il préférerait choisir $a>a^{*}$.

L'outil mathématique qui permet de choisir la bonne vitesse de convergence est la loi du logarithme itéré qui s'énonce comme suit.

Soit $\left\{y^{t}\right\}$ une suite de variables aléatoires indépendantes et de même loi avec une moyenne $\mu$ finie et une variance $\sigma^{2}$ finie. Alors pour tout $\lambda>1$

$$
\lim _{T} \sup \left[\left|\mu-\frac{1}{T} \sum_{t=1}^{T} y^{t}\right| /\left(\frac{2 \lambda \sigma^{2} \log \log T}{T}\right) \frac{1}{2}\right]<1
$$

avec probabilité 1 .

Lorsque les agents escomptent le futur (Radner [1985]) ou lorsque l'horizon est fini (Lambert [1983], Rogerson [1985 a], Henriet et Rochet [1986]), l'optimum d'information parfaite ne peut plus être réalisé et le contrat optimal réalise un partage du risque intertemporel en faisant dépendre l'allocation de la date $t$ de la suite d'événements passés (ce que l'on peut interpréter comme une justification des bonus-malus). 


\section{CONCLUSION}

J'ai choisi dans cette note d'être bref sur la théorie des contrats répétés pour deux raisons. D'une part, les résultats essentiels ne sont peut-être pas encore bien énoncés; d'autre part, pour pénétrer avec profit la littérature pertinente, il faut pouvoir se référer en détail à la théorie des jeux répétés, ce qui mérite un article spécifique. Je mentionnerai seulement quelques préoccupations actuelles supplémentaires.

Implicitement, nous avons supposé dans les développements ci-dessus que le principal peut s'engager non seulement sur une période comme nous l'avons toujours supposé, mais aussi pour tout l'avenir. Il peut, de façon crédible, proposer des contrats inter-temporels sans que l'agent ait à craindre qu'à une certaine date future le principal, au vue de l'information jusque-là accumulée, modifie le contrat.

Bien qu'on n'ait pas aujourd'hui de théorie de l'engagement crédible, divers auteurs explorent les conséquences de possibilités d'engagement limitées (voir, par exemple, Radner [1985], Radner, Myerson et Maskin [1986], Abreu, Pearce et Stachetti [1986]). Que ce soit avec engagement limité ou avec un taux d'escompte suffisamment faible, les résultats sont analogues à ceux obtenus arec engagement total et sans escompte, c'est-àdire qu'il est possible d'approcher l'optimum d'information parfaite ${ }^{1}$.

Les résultats sont toutefois très différents lorsqu'il y a plusieurs sources de risque moral ${ }^{2}$. Par exemple si à fois le principal et l'agent prennent des actions non observables ou si l'on étudie les équilibres de Nash dans une équipe où chaque agent choisit une action non observable, l'optimum d'information parfaite ne peut pas être restauré en général (voir Fudenberg et Maskin [1986]).

Nous sommes proche d'une compréhension assez satisfaisante de l'organisation constituée par un principal et un agent avec risque moral. De même, la structure des contrats optimaux en présence uniquement de sélection adverse est assez bien comprise (voir Laffont [1984], chap. 11, pour le cas statique, Baron et Besanko [1984] pour le cas dynamique avec engagement, Laffont et Tirole [1986 b] pour le cas dynamique sans engagement). Il subsiste encore un besoin de clarification pour les modèles avec à la fois risque moral et sélection adverse (Laffont

1. Notons aussi que Fama [1980] a suggéré dans le contexte particulier de la relation actionnaires-manager que le souci de sa carrière par le manager combiné à la force du marché des managers doit aussi restaurer i'optimum d'information parfaite. Toutefois comme l'a montré Holnström [1983], ceci est loin d'être évident car en général liaversion au risque et l'escompte du futur mettent une limite à l'aptitude du miarché à policier parfaitement les incitations.

2. Holmström [1981] avait tait remarquer que dans une organisation où tous les agentents contrôlent une variable de risque moral, l'impossibilité de s'engager de façon crédible à ne pas distribuer l'ensemble du produit commun - ce qui exclut des pénalités collectives - rend en général impossible l'optimum d'information imparfaite. Il y voyait même une justification pour le rôle d'un chef d'entreprise extérieur qui peut rendre crédible l'utilisation de pénalités collectives. 
et Tirole [1986 a], Melumad et Reichelstein [1984], Guesnerie, Picard et Rey [1986]). Ensuite, et l'effort est déjà entamé, il faudra s'attaquer aux organisations plus complexes avec, par exemple, un principal, un contrôleur et un agent (voir Tirole [1986]) dont l'aspect nouveau le plus important est la nécessité de tenir compte des phénomènes de coalitions entre certains individus (voir Green et Laffont [1979] pour l'étude des incitations pour les coalitions dans le cas de sélection adverse).

La voie est encore longue pour parvenir à une théorie économique des organisations satisfaisantes. Toutefois, grâce aux progrès de la théorie des incitations, les coûts de transaction liés aux asymétries d'information de type risque moral ou sélection adverse peuvent faire l'objet d'une évaluation qualitative qui permet de poser analytiquement des problèmes qu'on ne savait pas formaliser jusqu'à récemment. Il ne faut pas toutefois oublier que ces travaux laissent provisoirement à l'écart d'autres types de coûts de transaction et en particulier ceux liés aux coûts d'optimisation et aux contraintes de complexité. Même s'il existe certains travaux préliminaires (Reichelstein [1985], Green et Laffon [1986], Rubinstein [1986]), il s'agit là d'un défi intellectuel qui attend encore d'être relevé.

\section{RÉFÉRENCES BIBLIOGRAPHIQUES}

Abreu D., Pearce D., Stachetr E. [1986], "Optimal cartel equilibria with imperfect monitoring n, Journal of Economic Theory, 39, p. 251-269.

ARnow K. [1953], "Le rôle des valeurs boursières pour la répartition la meilleure des risques ", Cahiers du séminaire d'économétrie, 1.

Arrow K. [1963], * Uncertainty and the welfare economics of medical care », American Economic Review, 53, p. 941-973.

Baron D., BesanKo D. [1984], "Regulation and information in a continuing relationship x, Information Economics and Policy, 1, p. 447-470.

Borch K. [1962], " Equilibrium in a reinsurance market ", Econometrica, 30, p. 424-444.

FAMA E. [1980], a Agency problems and the theory of the firm ", Journal of Political Economy, 88.

Fudenberg D., Maskin E. [1986], "Discounted games with unobservable actions », miméo.

Green J., LAfFont J.-J. [1979], Incentives in public decision making, NorthHolland, Amsterdam.

Green J., LAfFont J.-J. [1986], "Incentive theory with data compression " (chap. 10) in Heuretr W., Starr R., Starrett D. [1986].

Green J., STOCKey N. [1983], "A comparison of tournaments and contracts n, Journal of Political Economy, p. 349-364.

Grossman S. J., Hart O. D. [1983], « An analysis of the principal-agent problem n, Econometrica, 51, p. 7-45.

Guesnerue R., LAfFont J.-J. [1978], a Taxing price makers ", Journal of Economic Theory, 19, p. 423-455.

Guesnerie R., Picard P., Rey P. [1986], "Adverse selection and moral hazard with risk neutral agent ", miméo.

Harrus M., Ravrv A. [1979], "Optimal incentive contracts with imperfect information n, Journal of Economic Theory, 20, p. 231-259. 
Het.xer W., Stark R., STARRETr D. [1986], Uncertainty, information and communication, Essays in Honor of K. J. Arrow, vol. III, Cambridge, Cambridge University Press.

HexwwrG M. [1983], " Moral hazard and monopolistically competitive insurance markets v, miméo.

Hedpman E., LAFFont J.-J. [1975], "On moral hazard in general equilibrium theory ", Journal of Economic Theory, 10, p. 8-23.

HENRUET D., Rocher J.-C. [1986], " La logique des systèmes bonus-malus en assurance automobile : une approche théorique ", Annales d'économie et de statistique, 1, p. 133-152.

Holmströм B. [1953], "Managerial incentives problems. A dynamic perspective ", miméo.

Hormström B. [1979], " Moral hazard and observability ", Bell Journal of Economics, 10, p. $71-91$.

Holmström B. [1981], "Moral hazard in teams ", miméo.

LAFFont J.-J. [1984], Cours de théorie micro-économique, vol. II : Economie de l'incertain et de l'information, Economica, Paris.

LafFont J.-J., TrRole J. [1986 a], "Using cost observability to regulate firms ", Journal of Political Economy, 94, p. 614-611.

Laffont J.-J., Tirole J. [1986 b], " The dynamics of incentive contracts », à paraître dans Econometrica.

LAMBERT R. [1983], a Long term contracts and moral hazard n, Bell Journal of Economics, 14, p. 41-452.

Lazear E., Rosen S. [1981], " Rank-order tournaments as optimum labor contracts n, Journal of Political Economy, 89, p. 811-864.

Melumad N., Rejcherstexn S. [1985], a Value of communication in principal-agent relationships $\#$, miméo.

Muraleses J. [1975], "The theory of moral hazard and unobservable behavior n, Part I, miméo.

MOOKHERJEE D. [1984], "Optimal incentive schemes in multi-agent situations \#, Review of Economic Studies, 51, p. 433-416.

NAlebufF B., Sricurrz J. [1983], " Prizes and incentives : towards a general theory of compensation and competition ", Bell Journal of Economics, p. 21-43.

PAUly M.V. [1968], "The economics of moral hazard : comment", American Economic Review, 58, p. 531-537.

RADNER R. [1981], " Monitoring cooperative agreements in a repeated principalagent relationship n, Econometrica, 49, p. 1127-1148.

RanNer R. [1985], "Repeated principal-agent games with discounting ", Econometrica, 53, p. 1173-1198.

Radner R., Myerson R., Masian E. [1986], " An example of a repeated partnership game with discounting and with uniformily inefficient equilibria , Review of Economic Studies, 53, p. 59-70.

RexCHELSteñ S. [1984], " Incentive compatibility and informational requirements n, Joumal of Economic Theory, 34, p. 32-51.

Rocerson W. [1985 a], « Repeated moral hazard n, Econometrica, 53, p. 69-76.

Rogerson W. [1985 b], a The first order approach to principal agent problems $m$, Econometrica, 53, p. 1357-1367.

Ross S. [1973], “The economic theory of agency. The principal's problem $\bowtie$, American Economic Review, 63, p. 134-139.

Rubinstedn A. [1979], a Offenses that may have been committed by accident. An optimal policy of retribution " in Brams S. J., Schottrer A., Schwödiaver G., eds., Applied game theory, Würzburg, Physica-Verlag.

Rubinsteja A., YaARi M.E. [1983], "Repeated insurance contracts and moral hazard , Journal of Economic Theory, 30, p. 74-97.

Rubinstenn A. [1986], "Finite automata play the repeated prisoner's dilemma ", Journal of Economic Theory, 39, p. 83-96.

Sraverl S. [1979], * Risk-sharing and incentives in the principal and agent relationship n, Bell Journal of Economics, 10, p. 55-73. 
Sxmon H. [1951], "A formal theory of the employment relationship ", Econometrica, 19, p. 293-305.

SPENCE M., ZeCKhaUSER R. [1971], "Insurance, information and individual action ", American Economic Review, 61, p. 380-387.

Srrcx.riz J. E. [1974], a Incentives and risk sharing in sharecropping n, Review of Economic Studies, 61, p. 219-256.

Tirole J. [1986], a Hierarchies and bureaucraties », miméo, MIT.

Wrason R. [1968], a The theory of syndicates n, Econometrica, 36, p. 119-132.

Zeckohauzer R. [1970], a Medical insurance : A case study of the trade-off between risk-spreading and appropriate incentives", Journal of Economic Theory, 2, p. $10-26$. 
